Journal of Computer Science 8 (8): 1235-1243, 2012

ISSN 1549-3636

(C) 2012 Science Publications

\title{
Application of Near Field Communication Technology for Mobile Airline Ticketing
}

\author{
Wayan Suparta \\ Institute of Space Science (ANGKASA), Universiti Kebangsaan Malaysia \\ 43600 UKM Bangi, Selangor Darul Ehsan, Malaysia
}

\begin{abstract}
Problem statement: Near Field Communication (NFC) technology opens up exciting new usage scenarios for mobile devices based platform. Users of NFC-enabled devices can simply point or touch their devices to other NFC-enabled elements in the environment to communicate with them ('contactless'), making application and data usage easy and convenient. Approach: The study describes the characteristics and advantages of NFC technology offers for the development of mobile airline ticketing. This scenario describes the potential to overcome the conventional systems that are not gated and use study tickets. In such a system, today a transport application can be loaded on a NFC-enabled phone. To study such a case, Yogyakarta International Airport was taken as an example for a discussion. Results: NFC technology which consisting of three modes of operation and with international standardization can be applied as contactless to simplicity transactions, content delivery and information sharing on a mobile based platform. Conclusion: The idea of NFC application for mobile airline ticketing has been discussed for Yogyakarta International Airport.
\end{abstract}

Key words: Near Field Communication (NFC), contactless, e-ticket, airline system, Yogyakarta International Airport, NFC application, content delivery and information

\section{INTRODUCTION}

Environment of departure terminal, either domestic or international in a country is a cultural reflection to the management system and the user terminal. Tickets are one important key in the process of departure where passengers need to go to certain destinations. Unfortunately, the tickets supposed to be available accountability, especially cheaper airline tickets are difficult obtained, mostly during the day of departure and at two weeks before and after the Eid celebration as well as peak season. To order a ticket, most of the passengers need to go to a kiosk, airport locket, ticket sales agents, or now it can be ordered online when the internet connection or $\mathrm{WiFi}$ is available. However, several issues are arisen if the tickets have been acquired such as the queues system at the counter check-in is unmanaged, the boarding process still requires an additional cost and all the processes are often repetitive, tedious and a waste of time. In short, the new breakthroughs need to be created and implemented to all sectors that require public services such as tax payments, electricity bills, bank loans and others. In other words, a new technology quietly taking shape on the horizon that could alter the use of consumer electronics has been established in 2004 to facilitate transactions, data exchange and connections with a touch (NFCF, 2011b), namely Near Field Communication (NFC). After co-invented by NXP
Semiconductors and Sony in 2002, it is expected to become a widely used system for making payments by smartphone around the world.

NFC is a short-range wireless communication protocol, which is primarily intended to be used on mobile phones (NFCF, 2011c). It is essentially as an extension of Radio Frequency Identification (RFID) technology, evolved from a combination of contactless identification and interconnection technologies provides data exchange between devices across distances of up to 10 centimeters. NFC enables rapid and easy communications. This short range is not a disadvantage, since it aggravates eavesdropping. Simply by bringing two NFC-enabled devices close together, they automatically initiate network communications without requiring the user to configure the setup; one device must have an NFC reader/writer and another must have an NFC tag. The tag is essentially an integrated circuit containing data, connected to an antenna that can be read and written by the reader. It operates within the globally available and unlicensed radio frequency Industry Scientific and Medical (ISM) band of $13.56 \mathrm{MHz}$ with supported data rates are currently 106, 212-424 Kbit/s. After formed in 2004 by NFC forum, NFC promotes sharing, pairing and transactions between NFC devices and develops and certifies device compliance with NFC standards (NFCF, 2011a). Payment and ticketing, service initiation and information sharing are the major use cases of this technology that can turn a mobile phone into a 


\section{J. Computer Sci., 8 (8): 1235-1243, 2012}

travel card, an event ticket or even a credit card. With these usage areas, NFC brings simplicity to transactions, content delivery and information sharing. Thus, it promises great opportunities to make people is lives easier. Furthermore, it provides new opportunities for mobile operators, banks, transport operators and merchants with faster transactions, less cash handling and new operator services. However, NFC remains as a field that number of researches done are limited.

The objective of this study is to discuss the characteristics and advantages of the NFC technology that can be implemented for public transport, especially airplane ticketing via mobile phone. This trend is supported by the enthusiastic over Indonesia mobile users, where Indonesia is the highest in Southeast Asian countries in using cell phones for communication. The only problem in behind of NFC development in Indonesia is that the technology still growing slowly. For example, the application for ticket payment and automated boarding process has not yet materialized. The constraint is the limited number of phones in the market with NFC chip and the high cost of adopting this technology. In addition, the mentality and the readiness of the airport infrastructure, providers and all stakeholders involved in the NFC ecosystem plays a crucial role in the success of this technology.

How NFC works: As introduced, NFC is a technology for contactless short-range communication. NFC-enabled communicates via magnetic field induction, where two loop antennas are located within each other's near field, effectively forming an air-core transformer. A signal supplier coupled to the antenna circuit to supply a drive signal to cause the antenna circuit to generate an RF signal. Most of the RF energy is concentrated in the allowed $14 \mathrm{kHz}$ bandwidth range, but the full spectral envelope may be as wide as $1.8 \mathrm{MHz}$ when using the ASK modulation. The frequency band $13.56 \mathrm{MHz}$ used is to first modulate a subcarrier and then use the subcarrier to modulate the main carrier. This result in a modulation product, generating two sidebands symmetrically at the frequencies between subcarrier and the main carrier, create a counteracting magnetic field according to Lenz's law. This implies that eddy currents cause a significant difference in the magnetic component
(B-field). Practically, the reader will induce a current in the transponder antenna coil, generates a magnetic field. On the other hand, it is designed to offer a more powerefficient and affordable alternative to Bluetooth for very short-range and low-bandwidth applications. It is also touted as providing a more robust, bandwidth-rich alternative to RFID and other "contactless" technologies.

There are two modes of operation covered by the NFC protocol: Active and passive. In active mode, both devices generate their own radio field to transmit data. In passive mode, only one device generates a radio field, while the other use load modulation to transfer data. The NFC protocol specified that the initiating device is responsible for generating the radio field in this case. The passive mode of communication is very important for battery-powered devices like mobile phones and PDAs that need to prioritize energy use. The NFC protocol enables such devices to be used in power-saving mode, so that energy can be conserved for other operations. In addition, the active and passive NFC modes can be used to describe the conditions needed to prevent collisions during initialization.

The number of short-range applications for NFC technology is growing continuously, appearing in all areas of life. Especially the use in conjunction with mobile phones offers great opportunities. Table 1 shows a quick comparison with other short-range communication technologies that currently be used (NFCF, 2011b; Ailisto et al., 2007).

International standards and co-operation: Because NFC is an open platform technology, NFC devices need to be standardized both in terms of standards and in terms of usage areas with specify the basic capabilities, such as the transfer speeds, the bit encoding schemes, modulation, the frame architecture and the transport protocol. After approved as an ISO/IEC standard in 2003, nokia, sony and philips as the leaders of the mobile phone market founded the NFC Forum in 2004. This organization develops the standards that define the overall architecture of the technology. The first standardized technology architecture and standards for NFC compliant device was introduced in June 2006.

Table 1: Comparing NFC to other close range communication technologies

\begin{tabular}{|c|c|c|c|c|}
\hline & NFC & RFID & IrDA & Bluetooth \\
\hline Setup time & $<0.1 \mathrm{~ms}$ & $<0.1 \mathrm{~ms}$ & $\sim 0.5 \mathrm{sec}$ & $\sim 6 \mathrm{sec}$ \\
\hline Range & Up to $10 \mathrm{~cm}$ & Up to $3 \mathrm{~m}$ & Up to $5 \mathrm{~m}$ & Up to $30 \mathrm{~m}$ \\
\hline Usability & $\begin{array}{l}\text { Human centric, easy, } \\
\text { intuitive, fast }\end{array}$ & Item centric, easy & Data centric, easy & Data centric, medium \\
\hline Selectivity & High, given, security & Partly given & Line of sight & Who are you? \\
\hline Use cases & $\begin{array}{l}\text { Pay, get access, share, } \\
\text { initiate service, easy set up }\end{array}$ & Item tracking & Control and exchange data & $\begin{array}{l}\text { Network for data exchange, } \\
\text { headset }\end{array}$ \\
\hline Consumer experience & Touch, wave and simply connect & Get information & Easy & Configuration needed \\
\hline
\end{tabular}




\section{J. Computer Sci., 8 (8): 1235-1243, 2012}

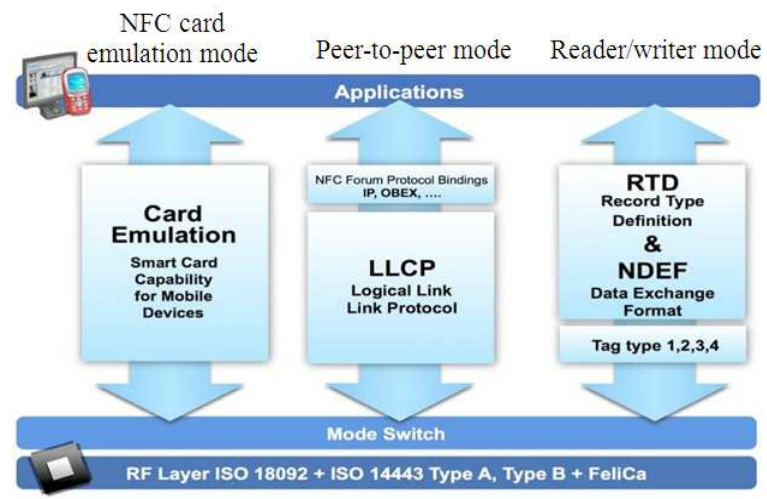

Fig. 1: Architecture of NFC technology defined by NFC Forum in 2011 (NFCF, 2011a)

Today this organization has over 150 members, including Microsoft, Samsung, Visa, MasterCard and HP. The proximity and vicinity standards are included as a base standard in Digital Protocol and Analogue specifications (ISO/IEC 14443, ISO/IEC 18092-NFCIP-1 and JIS X 6319-4-FeliCa) as well as ISO/IEC 15693.

Paus (2007) show that NFC is described by NFCIP-1 (Near Field Communication Interface and Protocol 1), which standardized in the following norms: ISO 18092, ISO 21481 and ECMA (340, 352 and 356) as well as ETSI TS 102 190. The technology is compatible with the international chip card standard ISO $14443 \mathrm{~A} / \mathrm{B}$, FeliCa and MIFARE infrastructures. Nowadays, NFC devices do not only implement NFCIP-1, but also NFCIP-2, which is defined in ISO 21481, ECMA 352 and ETSI TS 102 312. NFCIP-2 allows for selecting one of three operating modes (1) NFC data transfer (NFCIP-1), (2) Proximity Coupling Device (PCD), defined in ISO 14443 and (3) Vicinity Coupling Device (VCD) defined in ISO 15693. NFC devices have to provide these three functions in order to be compatible with the main international standards for smartcard interoperability, ISO 14443 (proximity cards, e.g., Philip's Mifare), ISO 15693 (vicinity cards) and to sonys felica contactless smartcard system. Hence, as a combination of smartcard and contactless interconnection technologies, NFC is compatible with today's field proven RFID-technology. It means that they provide compatibility with the millions of contactless smartcards and scanners that already exist around the world.

Operating modes: NFC operates in three different modes. Each of these modes requires that NFC devices use a common data format for communications as depicted in Fig. 1. With this mode, NFC technology can give additional functionality to a mobile device that can be used such as a contactless credit card or a contactless train ticket.
NFC Card Emulation (NCE) Mode: NFC devices can also act as smartcard (ISO 14443) and contain a secure smartcard chip also referred as a Secure Element (SE) that operates in card emulation mode. The secure element is connected to the NFC controller for proximity transactions (contactless payments). Host controller is able to exchange data with the secure element. A NFC-enabled device emulates a contactless payment card and can be used to purchase goods and services. In this mode, the device is passive so it does not generate a RF field.

Peer-to-Peer (P2P) Mode: In this mode (standardized in ISO/IEC 18092, ISO/IEC 14443-2,3,4 and JIS X6319-4), two devices communicate with each other and exchange data, such as passing contact information or an electronic business card from one device to another, for example, between a NFCenabled mobile phone and a NFC-enabled PC. There are three specifications in $\mathrm{P} 2 \mathrm{P}$ mode in order to determine the operating range of devices and testing the physical layer. Analogue Specifications defines the radio frequency characteristics of an NFC Forum device (e.g., shape and strength of RF fields). Digital protocol specification defines the building blocks for communication ("NFC Logical Link Control Protocol (LLCP)". NFC Activities Specification defines the activities required to set up communication in an interoperable manner, based on the building blocks of the digital protocol specification ("NFC Simple NDEF Exchange Protocol (SNPE)"), e.g., polling cycles, when to perform collision detection.

Reader/Writer (R/W) Mode: NFC requires two devices to communicate; one is NFC R/W in the format of the NFC data-exchange format (NDEF) and NFC Record-Type Definition (RTD) and the other an NFC tag. These two specifications will standardize how NFC devices operate in the $\mathrm{R} / \mathrm{W}$ mode. A NFC-enabled device can access data from a RFID-enabled object, such as a "smart poster" with an embedded RFID tag that allows users to download a URL for a movie trailer. NFC tags are passive devices that can be used to communicate with active NFC devices (an active NFC $\mathrm{R} / \mathrm{W}$ ). Message coding format used by NFC reference applications is transmitted by NDEF. It is allows multiple NDEF messages and allows messages to be divided into chunks such as phases, stages, activities, tasks and steps. In the RTD, they define how to construct records in NDEF messages. Records may contain other records. Each record has a type, indicating what it contains. Record types are extensible, they may be defined by NFC Forum specifications (reference applications) or by other bodies (using domain name). In order to communicate the NFC devices between the active NFC R/W and the passive NFC tag, four 
basic tag types have been defined that all NFC compatible devices must support:

- $\quad$ Tag type 1 (ISO/IEC 14443A): It has a 96 bytes memory capacity with communication speed of $106 \mathrm{Kbit} / \mathrm{s}$ (e.g., Innovision Topaz)

- $\quad$ Tag type 2 (ISO/IEC 14443B): It has a 48 bytes memory capacity with a communication speed of $106 \mathrm{Kbit} / \mathrm{s}$ (e.g., MIFARE Ultralight)

- Tag type 3 (Japanese Industrial Standard (JIS) X 6319-4, FeliCa): Its memory capacity is variable up to $1 \mathrm{MB}$ per service with a communication speed of $212 \mathrm{Kbit} / \mathrm{s}$ or $424 \mathrm{Kbit} / \mathrm{s}$. (e.g., Sony FeliCa) and

Tag type 4 (ISO/IEC 14443B series): Its memory capacity is variable up to 32 Kbytes per service with a communication speed up to $424 \mathrm{Kbit} / \mathrm{s}$ (e.g., MIFARE DESFire)

NFC Architecture in a mobile phone: Figure 2 shows the architecture of NFC technology in a mobile device defined by NFC Forum in 2011. As shown in Fig. 2a, NFC devices contain a Secure Element (SE), which theoretically composed of two components: A Java Smart Card and a Mifare card with a storage capacity of 4Kbytes. The SE is connected to the NFC controller for proximity transactions (contactless payments) through the Single-Wire Protocol (SWP) and is used for the tag emulation mode. Host controller is able to exchange data with the SE and allows setting the operating modes of the
NFC controller and process data sent and received. The architecture of the NFC device in a mobile phone is shown in Fig. 2b.

There is not yet any defined physical link between SE and NFC controller even though GSMA is appraising some options such as S2C (Signal- in Signalout connection) and SWP. As shown in Fig. 2b, the GSMA Mobile NFC device functional architecture has three major elements of a Java-based handset. On the below of the illustration is the NFC controller, Baseband processor and RF unit and there is the proximity antenna (similar to Fig. 1). NFC processor security connected to the SIM card through SWP in API NFC. On the middle of illustration, NCF applications could live in the SIM card connects to NFC processor using SWP (product by NXP), in Smart Cards or in a secure area in the handset memory. The application processor includes Runtime, APIs and NFC application. GMSA is pushing for the SIM card and handing the NFC ecosystem over to the network providers. The top of the illustration is the Java runtime and APIs for accessing SIM cards and contactless communication channels, as well as the Java MIDlet application. Note that the application consists of two parts, the MIDlet and a Java Card application that may reside on a SIM card or a smartcard and perhaps uses memory for the keystore. The three elements have been externally connected to the handset, which are readers, tags and smartcards with which the handset communicates with them.

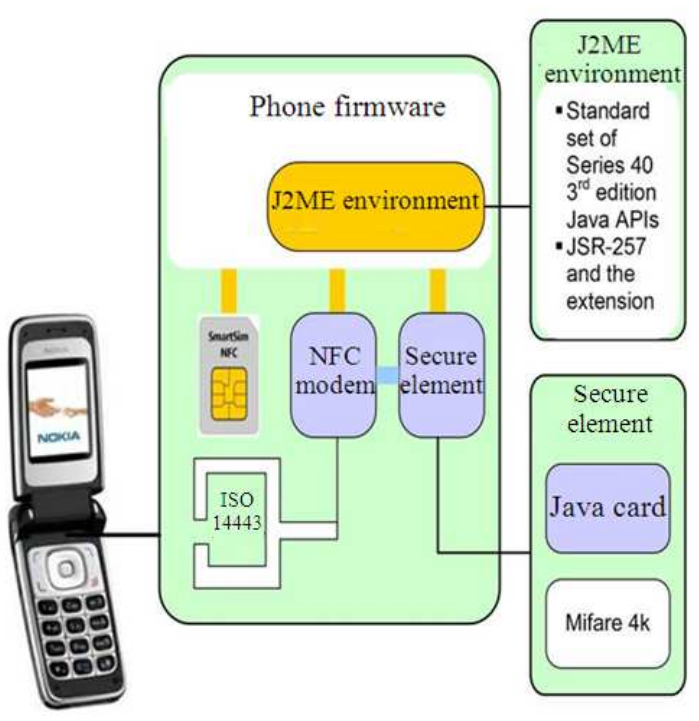

(a)

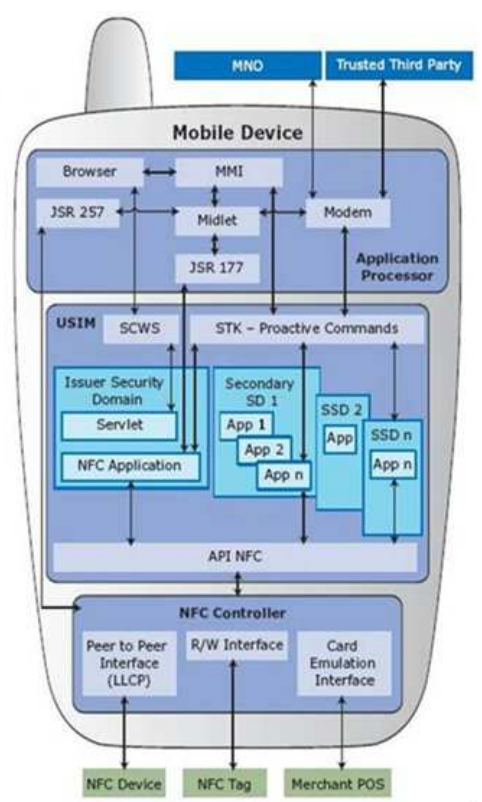

(b)

Fig. 2: (a) Architecture of NFC technology in a mobile device defined by NFCF in 2011 and (b) NFC integrated in GSMA (2011) 
From the technical viewpoint, the standardization of the technology is already advanced and the major technical decisions have been taken. Now it has rapidly evolved over the NFC-enabled handsets are commercially available. Hence, the greatest opportunity to realize this technology in various sectors of life is very promising.

\section{MATERIALS AND METHODS}

Designing of NFC for airplane mobile ticketing: One of the benefits of NFC technology, it can be used as a link that enables electronic ticketing or electronic payment, where tags contain ticket or e-money information and readers control this information to be processed as e-money or ticket. The technology has many advantages and it is fast replacing the other two types of ticketing. Some public transport networks are replacing their first ticketing generation system directly by a contactless one, omitting the magnetic ticket generation stage. Mobile ticketing using SMS (short text message) is a relatively quick and easy way to get a ticket issued to mobile phone users. A new system (model) that the ticket ordered via NFC mobile phone is come out with boarding pass as illustrated in Fig. 3 .

As shown in Fig. 3, the ticket selection is performed by sending an SMS to the background system (Trusted Service Manager (TSM) and Mobile Network Operator (MNO)), accompanied either by a specifying text or by sending it to a specific phone number for each possible ticket. An electronic ticket as a picture messaging is then returned via SMS, EMS, WAP Push, MMS or E-mail to the user. The first process before using NFC facilities needs an account registration to the operator and validates the account and payment method (credit cards, prepaid or postpaid bill phones). After complete a transaction, mobile tickets are being issued using SMS or mobile barcodes. When the ticket obtained, the next step is to store the ticket securely on the phone, each of which may have a different provider:

- A handset manufacturer can provide NFC-enabled phones with embedded secure elements

- SIM manufacturers can provide the SIM cards, via the mobile network operators, needed to store ticket applications

- Secure Digital card manufacturers can offer SD cards, when the ticket is stored in a pop-out card

- Travel itineraries are sent from the ticket server to the billing server

- The system reconstructs route and price and bills the customer
- The bill is transferred to the customer via e-mail. Overall process, the ticket in a 2D barcode form (following IATA role) was sent to mobile phone for electronic boarding passes

The next step after the ticket stored in the mobile phone is a check-in process ("contactless'). Figure 4 illustrates the check-in process for an airline ordered via NFC mobile phone. Note that the purchase size baggage can be selected and ordered during the ticket creation. System on the NFC mobile phone could work for baggage selection and prices and capable of direct payment of airport tax, so that the amount of airline tickets should contain of flight ticket, fuel surcharge, airport fee, passenger service fee. As shown in Fig. 4, logically the boarding process ('touch in') can be done with the following steps:

- Placing the device ('contactless') in front of the Touch Point and confirming

- Ticketing data is transferred from Touch Point to mobile phone

- The mobile phone transfer data to central of ticket server

- The central ticket serves issues the travel authorization and transmits data via mobile network to the cell phone. After confirmation/validation done, the oversize baggage can be send to bag drop counters, immigration check-in (for international flight) and waiting for boarding time in the waiting room for the last boarding step

- Deboarding ('touch out'), mobile phone transfer data to the ticket server. After that, run to the immigration point (for international passengers) and baggage claim

- The journey is finalized and the travel authorization on the mobile phone is reset

The minimum required infrastructure to provide easy use of the NFC-enabled phone is an NFC Forum Compliant tag. These are used for two things: Automatic start of the installed ticketing application or ticket purchased through a website (e.g., SMS ticket) and Authenticating the location (e.g., the location information can be stored on the tag). To purchase a ticket or to register a trip in the system, the passenger taps a NFC-enabled phone against the NFC Forum tag at the departure station. The tag provides data about the current location to the transport application or opens a dedicated website that then prompts the traveler to enter a destination. The destination can be selected either from a personalized list of preferred locations or entered with the phone's keyboard. 


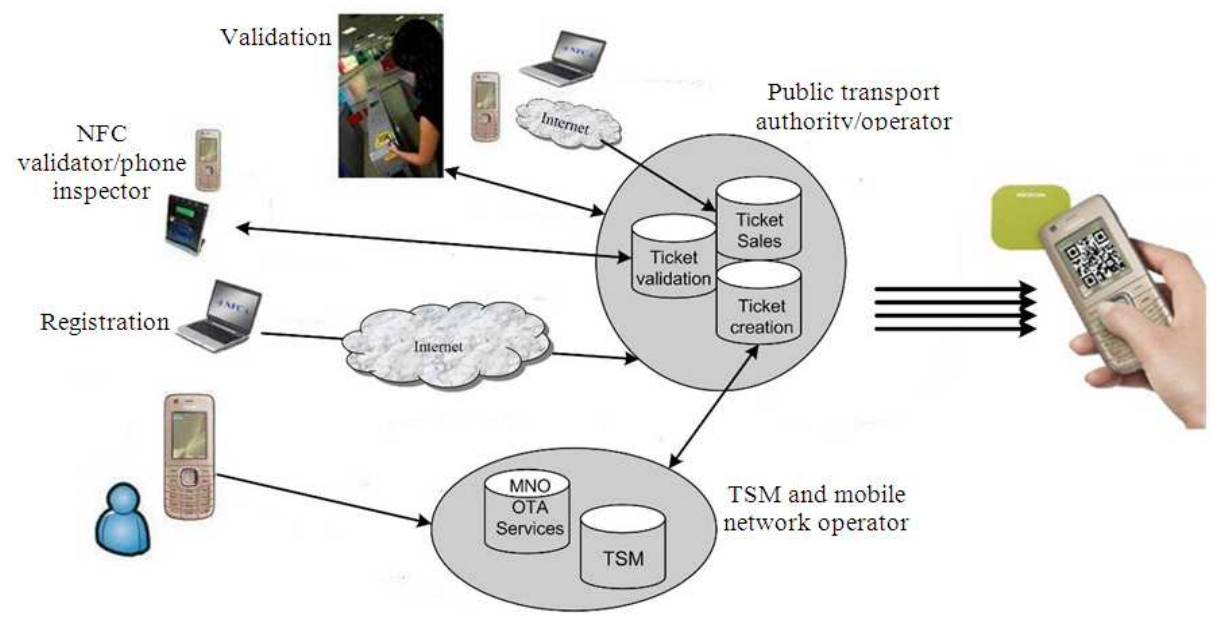

Fig. 3: Ticket ordered via mobile phone with boarding passes adapted from (NFCF, 2011c)

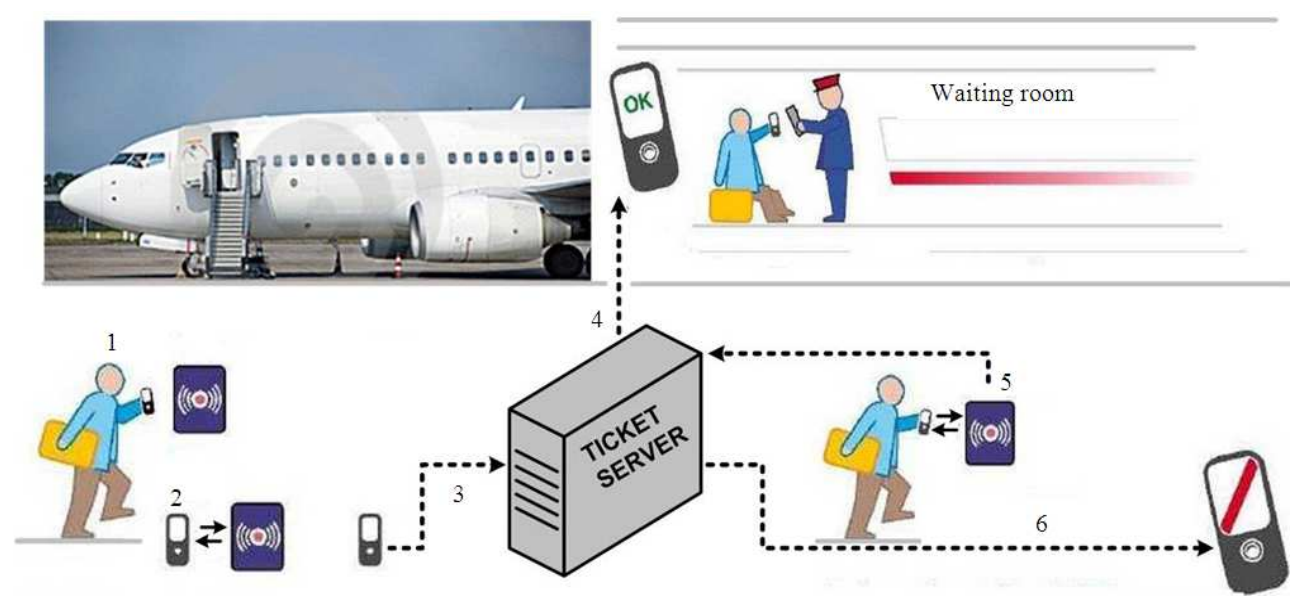

Fig. 4: Check-in and out processes using NFC-enabled mobile ticket

\section{RESULTS AND DISCUSSION}

A Case Study for Yogyakarta International Airport: The current problem raised with NFC technology is that not all handsets will be with a NFC chip. For ordering an airline ticket soon, one possibility is used the NFC sticker. NFC Sticker is backed by a Mifare Ultralight chip. It stores up to 48 bytes of user data, which can be made read-only. At present, Sagem Orga and Twinlinx (compliant with ISO 14443) had been presenting mobile contactless sticker powered by the Twinlinx "MyMax" and Sagem Orga Java Card" ${ }^{\mathrm{TM}}$ technologies Simply News, 2011. This interactive "SIMply Mobile Wallet" sticker adds NFC capability to existing and future mobile phones and it can be managed securely Over the Air (OTA). NFC Stickers can also be applied to smart phones to enable Google Wallet and NFC Payments as they already are in Japan. With this sticker, customer opts in the NFC check-in service to get the NFC sticker, then to install the NFC application to the handset. Customer pairs the NFC sticker with handset over the Bluetooth and configures the application with frequent flyer. If the mobile is ready, customer processes online check-in through airline. Figure 5 shows the detail of a check-in flowchart with NFC sticker handset.

With a capability of the NFC sticker on the handset or NFC chip embedded in the mobile, the next step in application of NFC technology for airline ticket is the provision of NFC readers. In this study, Adisucipto 
International Airport Yogyakarta is used as an example case study for application of NFC technology. Figure 6 shows the flowchart for check-in process without and with NFC technology.

Currently, a routine check-in at a normal process from departure to boarding and then the arrival steps in the same airport can be illustrated as in Fig. 6a. The crowded point is in the entrance (departure) and check-in queues. The situation is not due to the airport check-in area is too small for the international level. The most important is the organization and management systems include strict enforcement and the customer mentality during the queue. Therefore, with applied the NFC technology in this airport, the queue process and any additional payment can be reduced and finally, save the time and cost. As shown in Fig. 6b, check-in counter is only needed for bag drop because some of the customers will travel only with hand or carry-on luggage. If they have bagged drop or excess baggage, the NFC mobile boarding pass is also valid for baggage drop locations and clear payment. In addition, there is no need counter for airport tax payment. Airport taxes can be paid directly during the process of purchasing tickets. The ticket agent or e-airline ticket can directly be paying the customer tax to the airport management. So, in the public transport industry, e-ticketing systems are not only means of payment but with a huge amount of process information, which offers a large range of possibilities to make public transport easier to use, to manage and to control. Therefore, the long-term objective for the customer is to be able to pay for public transport without having to show or validate any card, relying on fully automatic fare payment.

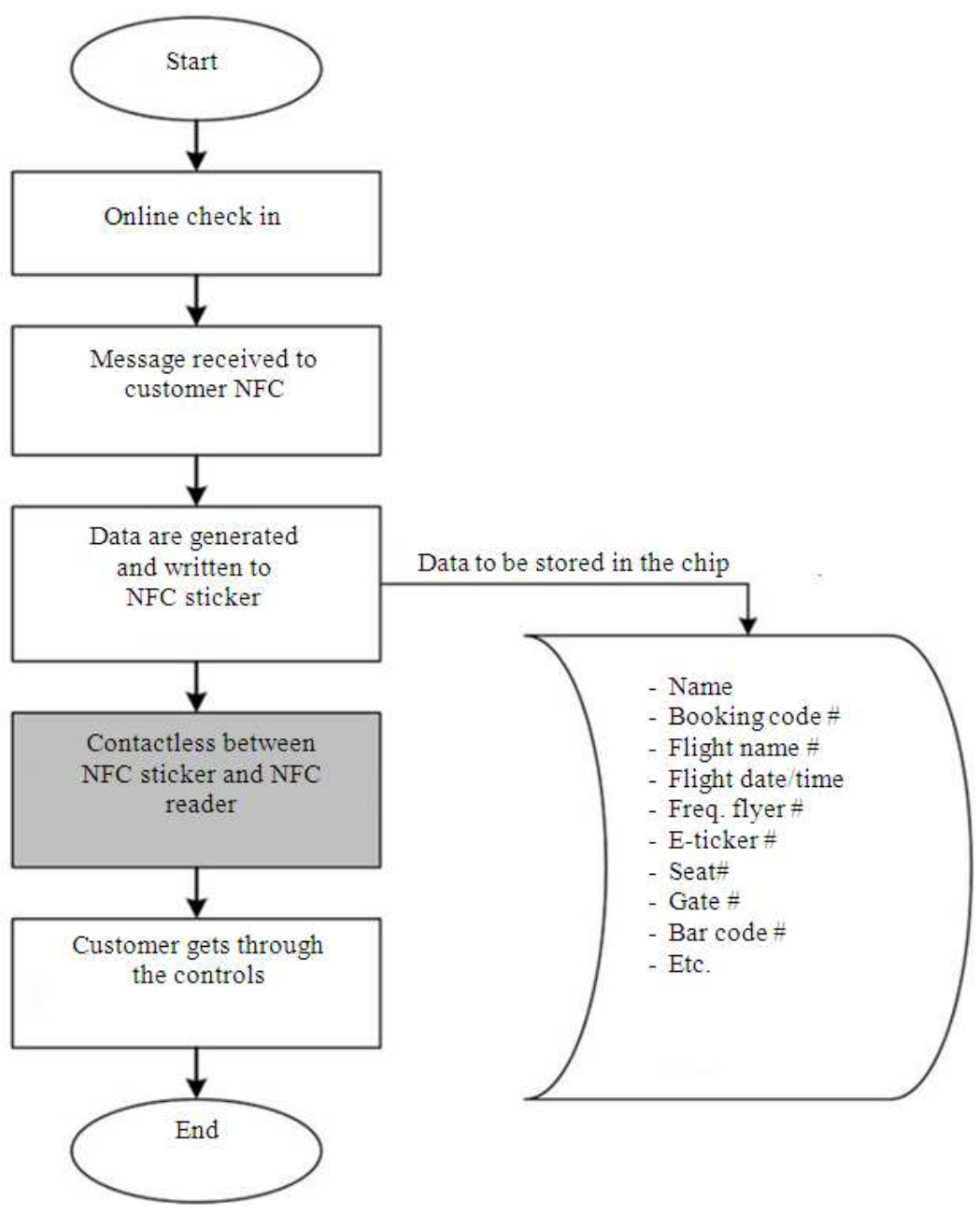

Fig. 5: Check-in process using NFC mobile sticker 


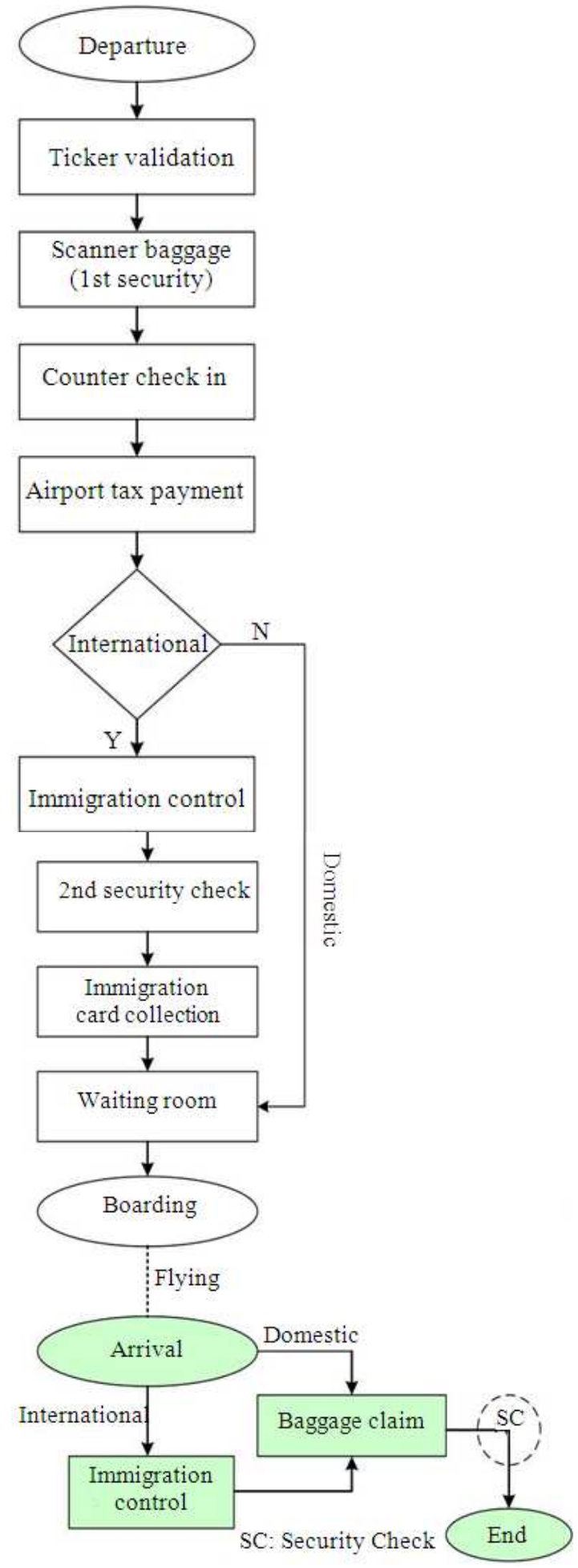

(a)

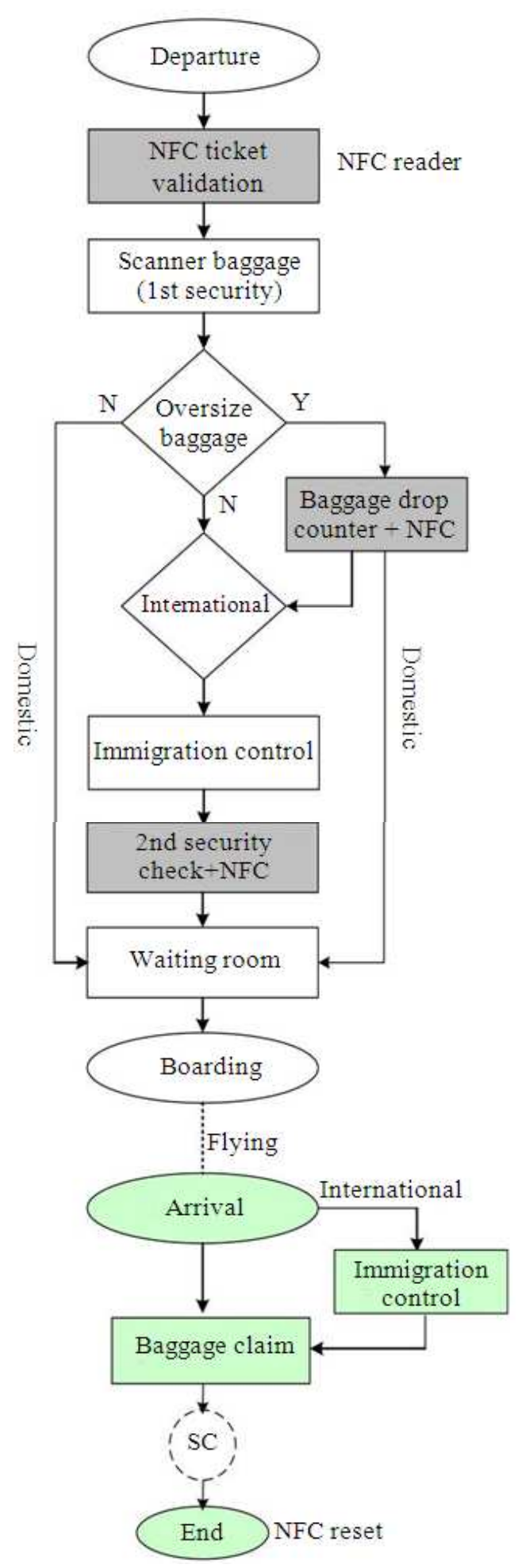

(b)

Fig. 6: Check-in and out processes for the case of Yogyakarta International Airport, (a) conventional and (b) proposed with NFC 


\section{CONCLUSION}

In this review, an example of the NFC application has been discussed. The discussion focused on the NFC technology, which will bring new perspective in all aspects of our life, especially for ticketing and mobile payment applications. With their progression, the NFC mostly targets application deals with privacy and wealth, usability and security risks. Hence, designing a secure system and user interface design strategies to be effective usable enough and appropriate for other types of system. Therefore, it is impossible not to realize the NFC technology in the Yogyakarta International Airport, at least at the new airport location in Kulon Progo, Yogyakarta as proposed by Yayasan Bendahara Yogyakarta. Specifically, it is opens up new application scenarios in improving services. For example, services at the university such as teaching and administrative, payment services in the university environment (e.g., student registration, university cafes and restaurants, library access, monitoring presence, university or faculty smart posters).

\section{REFERENCES}

Ailisto, H.M., T. Ylisaukko-Oja, A. Strommer, E. Hillukkala and M. Wallin et al., 2007. Physical Browsing with NFC technology. Valtion Teknillinen Tutkimuskeskus, Espoo.

GSMA, 2011. GSM association reference and device functional architectures. GSMA.

NFCF, 2011a. NFC Forum Technical Specifications. NFC.

NFCF, 2011b. What is NFC? NFCF.

NFCF, 2011c. NFC in Public Transport. 1st Edn., NFC Forum, Inc., USA., pp: 33.

Paus, A., 2007. Near field communication in cell phones. 